\title{
Application of a three-step approach for prediction of combustion instabilities in industrial gas turbine burners
}

\author{
Original article \\ Article history: \\ Accepted: 7 June 2017 \\ Published: 21 July 2017 \\ This paper is the updated version of a \\ paper originally presented at the 1st \\ Global Power and Propulsion Forum, \\ GPPF 2017, in Zurich, Jan 16-18 2017
}

\section{Check for updates}

*Correspondence:

GC: giovanni.campa@ansaldoenergia.com

Peer review:

Single blind

\section{Copyright:}

(c) 2017 lurashev et al. @ This is an open access article distributed under the Creative Commons Attribution Non Commercial No Derivatives License (CC BY-NC-ND 4.0). Unrestricted use, distribution, and reproduction of the original work are permitted for noncommercial purposes only, provided it is properly cited and its authors credited. No derivative of this work may be distributed.

\section{Keywords:}

combustion instabilities; time-domain analysis; technically premixed flame

\section{Citation:}

lurashev D., Campa G., Anisimov V. V., Cosatto E., Rofi L., and Bertolotto E. (2017). Application of a three-step approach for prediction of combustion instabilities in industrial gas turbine burners. Journal of the Global Power and Propulsion Society. 1: $129-141$.

https://doi.org/10.22261/JCW78T
Dmytro lurashev ${ }^{1}$, Giovanni Campa 2,*, Vyacheslav V. Anisimov², Ezio Cosatto ${ }^{2}$, Luca Rofi ${ }^{2}$, Edoardo Bertolotto ${ }^{2}$

${ }^{1}$ Department of Civil, Chemical and Environmental Engineering (DICCA), University of Genoa, Via Montallegro, 1, 16145 Genoa, Italy

${ }^{2}$ R\&D/Combustor, Ansaldo Energia S.p.A., Via Nicola Lorenzi, 8, 16152 Genoa, Italy

\begin{abstract}
Recently, because of environmental regulations, gas turbine manufacturers are restricted to produce machines that work in the lean combustion regime. Gas turbines operating in this regime are prone to combustion-driven acoustic oscillations referred as combustion instabilities. These oscillations could have such high amplitude that they can damage gas turbine hardware. In this study, the three-step approach for combustion instabilities prediction is applied to an industrial test rig. As the first step, the flame transfer function (FTF) of the burner is obtained performing unsteady computational fluid dynamics (CFD) simulations. As the second step, the obtained FTF is approximated with an analytical time-lag-distributed model. The third step is the time-domain simulations using a network model. The obtained results are compared against the experimental data. The obtained results show a good agreement with the experimental ones and the developed approach is able to predict thermoacoustic instabilities in gas turbines combustion chambers.
\end{abstract}

\section{Introduction}

Nowadays, gas turbine manufacturers have to meet ecological requirements, particularly reduction of $\mathrm{NO}_{\mathrm{X}}$ emissions. The most common way to meet these ecological requirements is to produce gas turbines that operate in the lean combustion regime. However, operation in the lean combustion regime makes the combustion system more prone to the occurrence of combustion instabilities (Lieuwen and Yang, 2005; Huang and Yang, 2009; Sirignano, 2015), which may cause heavy damages. This requires prevention of combustion instabilities which, in turn, requires an understanding of the nature of their onset.

There are different methods and numerical tools for the prediction of combustion instabilities. The numerical tools used for thermoacoustic analysis could be divided into two 
large groups: frequency-domain analyses (Nicoud et al., 2007; Camporeale et al., 2011) and timedomain simulations (Pankiewitz and Sattelmayer, 2003; Li and Morgans, 2015). The first type of analysis is usually used in the linear analysis, i.e., to predict whether the setup is stable or not (Camporeale et al., 2011). It can also be used to predict the amplitude of unstable pressure fluctuations. To accomplish this task, Silva et al. (2013) propose to perform simulations for each amplitude of acoustic oscillations that is characterised by its own FTF. The value of acoustic oscillation amplitude that corresponds to the zero growth rate is said to be the amplitude of saturated oscillations. The procedure requires performing a set of several simulations with different FTFs. On the contrary, using the time-domain analysis, both eventual unstable frequencies and their amplitudes are computed straightforwardly.

The straightforward way of the time-domain analysis of thermoacoustic instabilities would be performing unsteady CFD simulations of processes in gas turbine chambers with full complex geometries. Continuous improvement of computational capabilities of high-performance computing clusters makes this task possible (Poinsot and Veynante, 2005; Staffelbach et al., 2009) but still computationally expensive. Thermoacoustic phenomena could be decoupled in processes of chemical reactions (combustion), turbulence and acoustics. Decoupling of turbulent combustion and acoustics is artificial, but it helps to simplify the analysis. Acoustic length and time scales are often considered to be much larger than chemical and turbulent scales. This makes possible to perform simulations of turbulent combustion and acoustics separately in two different tools.

First, we compute the response of the flame to low-amplitude acoustic excitations with the help of large eddy simulations (LES) using the thickened flame model of the AVBP code. As a result, the FTF (Schuller et al., 2002; Kim et al., 2011) of the setup is computed. Then, the obtained FTF is approximated with an analytical time-lag-distributed model.

Finally, we use a simplified wave-based approach implemented in Simulink to find the stability margins of parameters under which gas turbines could be operated without going into self-excited pressure oscillations. Li and Morgans (2015) have shown that thermoacoustic simulations in the timedomain using the wave-based approach with nonlinear flame model could predict different nonlinear behaviours of the system. The strong feature of the network model simulations in the time domain is that it is possible to predict the unstable frequencies of the system just knowing the FTF. And it is possible to predict the amplitude of these oscillations as soon as the flame describing function (FDF) - the response of the flame to velocity perturbations of various amplitudes (Palies et al., 2011) — is found.

In this study, we discuss an atmospheric single-burner test rig developed by Ansaldo Energia (AEN) and Centro Combustione Ambiente (Rofi et al., 2015) equipped with one full-scale AEN gas turbine burner. Dependence of thermoacoustic stability of the setup on several parameters is discussed.

The article is structured as follows. First, the theoretical background of the three-step approach is explained. Second, the FTF calculated by LES is presented. Third, the calculated FTF is approximated by the time-lag-distributed model. Then, both linear and weakly nonlinear calculations are performed and the results are compared to the experimental ones. Finally, conclusions are given in the last section.

\section{Background}

\section{Description of the large eddy simulations software for flame transfer function calculations}

Recently, LES has been used to perform simulations of self-excited thermoacoustic instabilities (Poinsot and Veynante, 2005; Staffelbach et al., 2009) and to perform forced simulations to determinate the FTF (Poinsot and Veynante, 2005; Wolf et al., 2012). In this article, results of LES, particularly FTF, described by Rofi et al. (2015) are used. To improve reading of the article, the description of LES is provided. The reactive multi-species Navier-Stokes equations on unstructured grids are solved using a fully compressible explicit code AVBP (Schønfeld and Rudgyard, 1999). 
The viscous stress tensor, the heat diffusion vector and the species molecular transport use gradient approaches. The fluid viscosity follows the Sutherland law and the species diffusion coefficients are obtained using a constant specie Schmidt number and diffusion velocity corrections for mass conservation (Hermeth et al., 2013). A high-order finite element scheme is used for both time and space discretisation. The turbulent stress term is modelled by the classical Smagorinsky model (Smagorinsky, 1963). A chemical mechanism with six species $\left(\mathrm{CH}_{4}, \mathrm{O}_{2}, \mathrm{CO}_{2}, \mathrm{CO}, \mathrm{H}_{2} \mathrm{O}\right.$ and $\mathrm{N}_{2}$ ) and three reactions (Selle et al., 2004)

$$
\begin{gathered}
2 \mathrm{CH}_{4}+3 \mathrm{O}_{2} \rightarrow 2 \mathrm{CO}+4 \mathrm{H}_{2} \mathrm{O} \\
2 \mathrm{CO}+3 \mathrm{O}_{2} \rightarrow 2 \mathrm{CO}_{2} \\
2 \mathrm{CO}_{2} \rightarrow 2 \mathrm{CO}+3 \mathrm{O}_{2}
\end{gathered}
$$

is used to model methane/air combustion. The dynamic thickened flame model (Colin et al., 2000) is adopted to describe the iteration between turbulence and chemistry.

Usage of low-Mach number or "incompressible LES" (Han et al., 2015) can give the advantage of shorter calculation times. Compressible LES are performed in this study, because in compressible LES, fewer approximations are made, thus they give more precise results.

\section{Time-lag-distributed model for flame transfer function}

In general, heat release rate $\mathrm{Q}$ oscillations of technically premixed swirl-stabilised flames depend on perturbations of velocity $u$ and equivalence ratio $\varphi$ (Huber and Polifke, 2009a)

$$
\begin{aligned}
& \frac{\widehat{Q}_{u}(\omega)}{\bar{Q}}=F T F_{u}(\omega) \frac{\widehat{u}(\omega)}{\bar{u}}, \\
& \frac{\widehat{Q}_{\varphi}(\omega)}{\bar{Q}}=F T F_{\varphi}(\omega) \frac{\widehat{\varphi}(\omega)}{\bar{\varphi}} .
\end{aligned}
$$

First, let us consider the response of the flame to upstream velocity perturbations. Once longitudinal acoustic wave reaches a burner swirler, it is divided into two components: oscillations of axial velocity and oscillations of tangential velocity. Komarek and Polifke (2010) have shown that the response of perfectly premixed swirl-stabilised flame to velocity fluctuations depends on both oscillations of two components of velocity — axial and tangential — and have proposed the time-lag-distributed model of the flame response to upstream velocity fluctuations.

$$
\operatorname{FTF}_{u}^{\text {mod }}(\omega)=e^{-i \omega \tau_{1}-0.5\left(\omega \sigma_{1}\right)^{2}}+e^{-i \omega \tau_{2}-0.5\left(\omega \sigma_{2}\right)^{2}}-e^{-i \omega \tau_{3}-0.5\left(\omega \sigma_{3}\right)^{2}},
$$

where $\tau_{i}$ is the time delay of the corresponding mechanism, $\sigma_{i}$ is the standard deviation of the corresponding time delay. The response of the flame to the axial perturbations of the velocity is modelled with the parameters $\tau_{1}$ and $\sigma_{1}$. The parameters $\tau_{2}, \sigma_{2}, \tau_{3}$ and $\sigma_{3}$ model the response of the heat release to the tangential perturbations of the velocity produced by a swirler.

The physical meaning of the parameters $\tau_{i}$ and $\sigma_{i}$ is understood if we switch from the frequencydomain representation of the FTF to its time-domain representation, i.e., to the unit impulse response (UIR). The UIR in this study is the response of the normalised heat release to the normalised velocity perturbation of unit amplitude. The analytical form of the UIR corresponding to the FTF of Equation (4) is

$$
U I R_{u}^{\bmod }(t)=\frac{1}{\sigma_{1} \sqrt{2 \pi}} e^{-\frac{1}{2}\left(\frac{t-\tau_{1}}{\sigma_{1}}\right)^{2}}+\frac{1}{\sigma_{2} \sqrt{2 \pi}} e^{-\frac{1}{2}\left(\frac{t-\tau_{2}}{\sigma_{2}}\right)^{2}}-\frac{1}{\sigma_{3} \sqrt{2 \pi}} e^{-\frac{1}{2}\left(\frac{t-\tau_{3}}{\sigma_{3}}\right)^{2}} .
$$

Thus, Equation (5) models the response of the heat release to acoustic oscillations with three Gaussians with mean values $\tau_{i}$ and standard deviations $\sigma_{i}$. The first Gaussian in Equation (5) described with pair of parameters $\tau_{1}, \sigma_{1}$ models the time that fluid particles spend to travel from the zones where the flame is anchored to different points at the flame. Parameters $\tau_{2}, \sigma_{2}, \tau_{3}$ and $\sigma_{3}$ model the time that a fluid particle spends to travel from the swirler to the flame. 
Albayrak et al. (2015) have shown that the equivalence ratio fluctuations influence the heat release through three mechanisms: change of the heat of reaction, change of the flame speed and change of the flame surface area. It can be seen from Figure 4 of Huber and Polifke (2009b) that the response of the turbulent flame to equivalence ratio fluctuations could be modelled similarly to Equation (4) and Equation (5).

$$
\begin{gathered}
\operatorname{FTF}_{\varphi}^{\bmod }(\omega)=e^{-i \omega \tau_{4}-0.5\left(\omega \sigma_{4}\right)^{2}}+e^{-i \omega \tau_{5}-0.5\left(\omega \sigma_{5}\right)^{2}}-e^{-i \omega \tau_{6}-0.5\left(\omega \sigma_{6}\right)^{2}}, \\
\operatorname{UIR}_{\varphi}^{\bmod }(t)=\frac{1}{\sigma_{4} \sqrt{2 \pi}} e^{-\frac{1}{2}\left(\frac{t-\tau_{4}}{\sigma_{4}}\right)^{2}}+\frac{1}{\sigma_{5} \sqrt{2 \pi}} e^{-\frac{1}{2}\left(\frac{t-\tau_{5}}{\sigma_{5}}\right)^{2}}-\frac{1}{\sigma_{6} \sqrt{2 \pi}} e^{-\frac{1}{2}\left(\frac{t-\tau_{6}}{\sigma_{6}}\right)^{2}},
\end{gathered}
$$

where parameters $\tau_{4}, \sigma_{4}, \tau_{5}$ and $\sigma_{5}$ together model the flame response to change of the heat of reaction and change of the flame speed, and parameters $\tau_{6}$ and $\sigma_{6}$ model the flame response to area changes. Parameters $\tau_{4}, \sigma_{4}, \tau_{5}, \sigma_{5}, \tau_{6}$ and $\sigma_{6}$ model the time that fluid particle spends to travel from the point of the fuel injection to different points at the flame.

Many gas turbines burners, including the burner under consideration, are designed in such way that the pressure drop of the gas through the burner is around one order of magnitude higher than the pressure drop of the air. In this case, the acoustic perturbations in the gas turbine combustion chamber excite oscillations of the air mass flow through the burner; meanwhile, the gas mass flow remains almost unperturbed. This allows to make an assumption that the equivalence ratio $\varphi$ perturbations depend only on the air flow velocity $u$ fluctuations in the burner (Schuermans et al., 2004)

$$
\frac{\varphi^{\prime}}{\bar{\varphi}}=-\frac{u^{\prime}}{\bar{u}} .
$$

For such burners, it is possible to write a model for the total FTF.

$$
\operatorname{FTF}_{t o t}^{\text {mod }}(\omega)=\operatorname{FTF}_{u}^{\text {mod }}(\omega)-\operatorname{FTF}_{\varphi}^{\text {mod }}(\omega),
$$

where the fluctuations of the flame heat release rate $\mathrm{Q}^{\prime}$ depend only on the velocity fluctuations $u^{\prime} r$ at a reference position $r$ upstream of the flame

$$
\frac{\widehat{Q}(\omega)}{\bar{Q}}=F T F_{t o t}^{\bmod }(\omega) \frac{\hat{u}_{r}(\omega)}{\bar{u}_{r}} .
$$

At this point, the model for the FTF consists of 12 parameters and it could be cumbersome to determine all of them. However, for burners that have the fuel injection at the swirler blades, the values of the characteristic time delays modelled by parameters $\tau_{4}$ and $\tau_{6}$ are close to the values of the characteristic time delays modelled by parameters $\tau_{2}$ and $\tau_{3}$, respectively, and they cancel the resulting effect of each other. Thus, the model in Equation (10) can be further simplified using only four parameters (Schuermans et al., 2004; Ćosić et al., 2014).

$$
\begin{aligned}
& \text { FTF }_{\text {tot, simpl }}^{\text {mod }}(\omega)=e^{-i \omega \tau_{1}-0.5\left(\omega \sigma_{1}\right)^{2}}-e^{-i \omega \tau_{2}-0.5\left(\omega \sigma_{2}\right)^{2}}, \\
& U I R_{\text {tot, simpl }}^{\text {mod }}(t)=\frac{1}{\sigma_{1} \sqrt{2 \pi}} e^{-\frac{1}{2}\left(\frac{t-\tau_{1}}{\sigma_{1}}\right)^{2}}-\frac{1}{\sigma_{2} \sqrt{2 \pi}} e^{-\frac{1}{2}\left(\frac{t-\tau_{2}}{\sigma_{2}}\right)^{2}} .
\end{aligned}
$$

Two Gaussians described by Equations (11) and (12) model in a simplified way the change of the heat release due to perturbations of the velocity themselves and due to perturbations of equivalence ratio, respectively. They have the same relative amplitudes because increasing the velocity of the flow by certain relative value or decreasing equivalence ratio by the same relative value would increase heat release by the same relative value.

The FTF model described by Equation (11) is used further in this study.

\section{Wave-based approach for thermoacoustic simulations}

When the length of the setup under consideration is much larger than its dimensions in the other directions, it is possible to perform a one-dimensional low-order acoustic analysis (Bellucci et al., 2005; Schuermans et al., 2010; Bothien et al., 2015; Iurashev et al., 2016b). 
The test rig is considered as a set of sections with constant cross-sectional area. Pressure, velocity, temperature and density are decomposed into the sum of their mean component (denoted by ${ }^{-}$) and their fluctuating component (denoted by '). Mean values of pressure, velocity, temperature, density and thermophysical properties are assumed to be constant along each section and are changing only from section to section.

Perturbations of pressure $p$, velocity $u$ and density $\rho$ in each section could be represented in terms of downstream and upstream propagating acoustic waves (characteristics) (see Figure 1):

$$
\begin{gathered}
p^{\prime}(x, t)=f\left(t-\frac{x}{\bar{c}_{s}+\bar{u}}\right)+g\left(t+\frac{x}{\bar{c}_{s}-\bar{u}}\right), \\
u^{\prime}(x, t)=\frac{1}{\bar{\rho} \bar{c}_{s}}\left[f\left(t-\frac{x}{\bar{c}_{s}+\bar{u}}\right)-g\left(t+\frac{x}{\bar{c}_{s}-\bar{u}}\right)\right], \\
\rho^{\prime}(x, t)=\frac{1}{\bar{c}_{s}^{2}}\left[f\left(t-\frac{x}{\bar{c}_{s}+\bar{u}}\right)+g\left(t+\frac{x}{\bar{c}_{s}-\bar{u}}\right)\right],
\end{gathered}
$$

where $f$ and $g$ are the downstream and upstream travelling components (Riemann invariants) of acoustic waves, respectively, and $\bar{c}_{s}$ is the mean speed of sound in the section.

In order to connect oscillating variables in different sections (see Figure 2), we need to know the jump conditions. To compute the jump conditions between sections separated by a compact acoustic element, such as sharp cross-sectional area change and a compact swirler, the system of linearised equations of conservation of mass and Bernoulli (Dowling and Stow, 2005; Iurashev et al., 2016b) written in terms of $f$ and $g$ is solved.

$$
\mathbf{F}\left[\begin{array}{l}
f_{d} \\
g_{u}
\end{array}\right]=\mathbf{K}\left[\begin{array}{l}
f_{u} \\
g_{d}
\end{array}\right],
$$

where subscripts $u$ and $d$ denote the upstream and downstream sections, respectively. Matrices $\mathbf{F}$ and $\mathbf{K}$ take into account acoustic losses between sections; their coefficients can be found in Appendix A.

To calculate the jump conditions at the flame, the system of linearised equations of conservation of momentum and energy (product of mass conservation equation and Bernoulli equation) written in terms of $f$ and $g$ is solved (Dowling and Stow, 2005; Iurashev et al., 2016b).

$$
\mathbf{J}\left[\begin{array}{c}
f_{d} \\
g_{u}
\end{array}\right]=\mathbf{H}\left[\begin{array}{c}
f_{u} \\
g_{d} \\
Q^{\prime}
\end{array}\right],
$$

where coefficients of matrices $\mathbf{J}$ and $\mathbf{H}$ can be found in Appendix A. Equations (16) and (17) are directly used in the network model time-domain simulations.

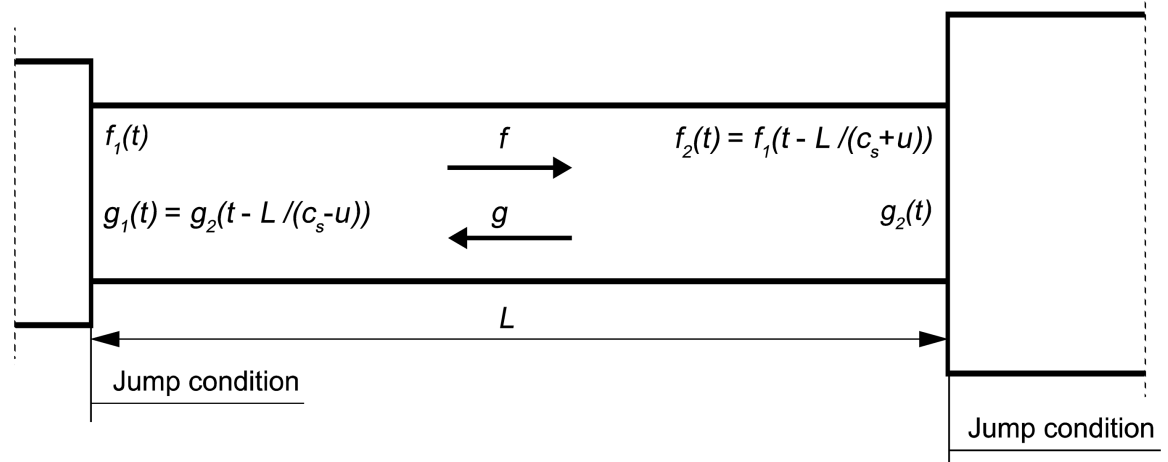

Figure 1. Scheme of waves propagation in a section of a low-order acoustic model. 


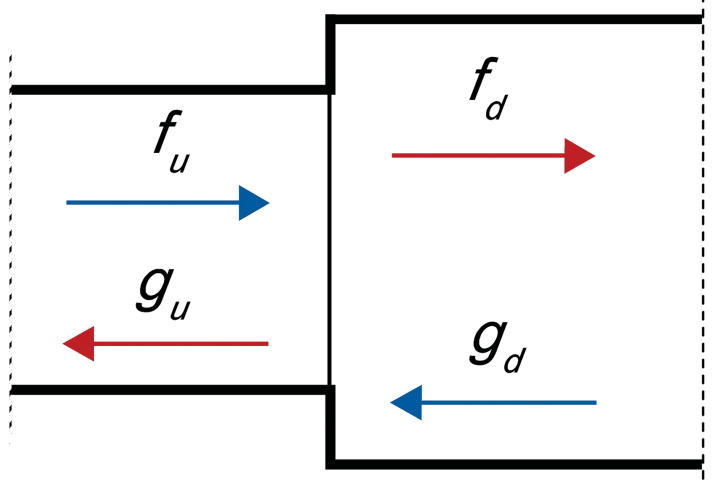

Figure 2. Scheme of waves propagation between sections of a low-order acoustic model.
At the beginning of the first section and at the end of the last section, $f$ and $g$ waves are related by the reflection coefficients $R_{\text {inlet }}$ and $R_{\text {outlet }}$, respectively.

There are four main sources of acoustic losses: at cross-sectional area changes, at partially reflective boundaries, at acoustic boundary layers and due to viscose dissipation. In this study, only the first two mechanisms of acoustic losses are taken into account.

Since the network model is implemented in Simulink, it is possible to design and test both active and passive dampers of thermally driven acoustic oscillations in future work.

\section{Step 1. Modelling the flame transfer function}

\section{Description of the experimental setup}

The atmospheric test rig is characterised by two cylindrical volumes, the plenum and the combustion chamber, connected to each other by a full-scale industrial burner. A schematic view of the geometrical setup utilised for the thermoacoustic experimental campaign is shown in Figure 3.

A thick layer of refractory is interposed between the chamber walls and the external liner to make the test rig adiabatic. During the experimental session, it is possible to control air and fuel mass flow rate, as well as air temperature and the length of plenum and chamber. The length of the rig can be continuously varied in order to tune the frequency at which combustion instabilities occur. Further details about experimental setup can be found in the study by Rofi et al. (2015).

\section{Description of the numerical setup}

The LES computational domain is formed by a fully unstructured mesh of 11852789 tetrahedral elements. The mesh is refined in correspondence of the flame and mixing regions; the time step is $4 \times 10^{-8} \mathrm{~s}$, resulting in a Courant number equal to 0.7 .

In order to reduce the computational costs and to be able to use a finer mesh in the flame region, the LES domain is shorter than the real combustion chamber (about one third of the real length). This assumption is considered acceptable since the recirculation zones are located well inside the LES domain; the flame is located in the first third of the LES domain; there is no diameter variation inside the test rig.

Inlet and outlet boundary conditions are imposed using the non-reflecting Navier-Stokes characteristic boundary condition formulation to control acoustic reflection (Poinsot and Lele, 1992). All the other walls are set as adiabatic and modelled using a logarithmic wall-law condition.

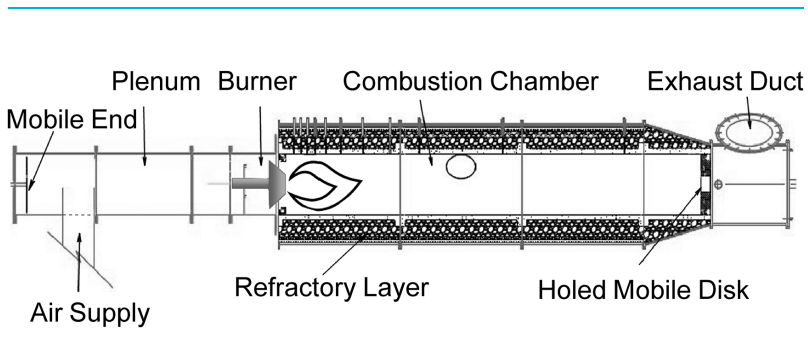

Figure 3. Scheme of the experimental test rig (Laera and Camporeale, 2017).

\section{Flame transfer function numerical calculation}

Once LES of the reactive process is statistically converged, a specific procedure to compute the FTF is performed. A multisinusoidal signal is imposed as velocity component normal to the burner inlet (Rofi et al., 2015). This signal is applied both at the diagonal and at the axial inlet to excite heat 

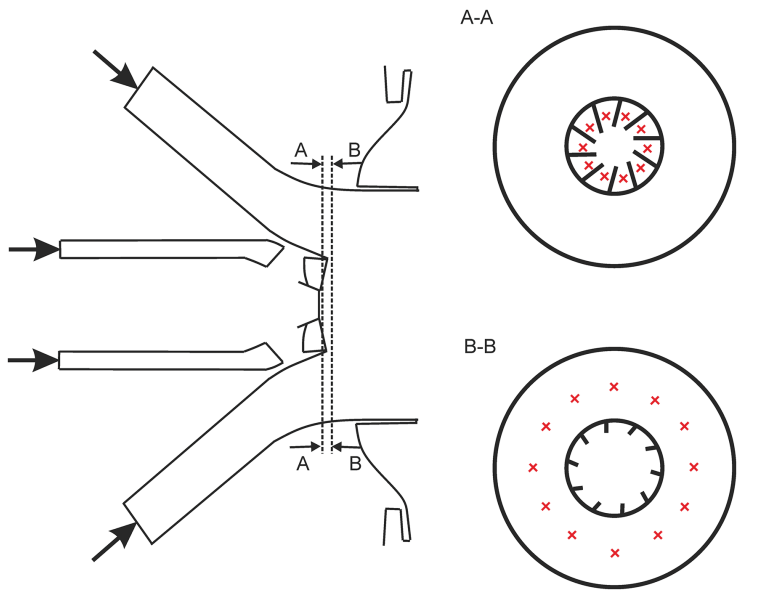

Figure 4. Schematic probes position for the FTF calculation (Rofi et al., 2015). tion chamber. The simulation is run for a time to guarantee at least six periods of oscillations at the lowest frequency of excitation. Frequencies are reported in terms of Strouhal number, which is calculated taking the inner diameter of the combustion chamber and the average velocity at the exit of the burner as the reference length and velocity, respectively. The FTF is calculated for each frequency imposed in the multi-sinusoidal signal and is shown in Figure 5.

\section{Step 2. Analytical model for the flame transfer function}

Optimum values of parameters $\tau_{i}$ and $\sigma_{i}$ of the FTF modelled with Equation (11) are computed approximating the FTF calculated with LES using the method of least squares. Values of $\tau_{i}$ and $\sigma_{i}$ normalised with respect to mean flow velocity at the burner exit and the diameter of the combustion chamber are presented in Table 1 and the corresponding model of the FTF is shown in Figure 5.

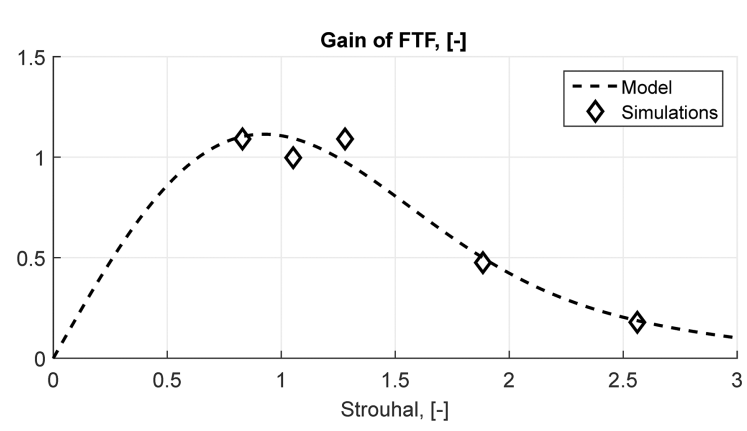

\section{Step 3. Stability analysis using wave- based approach}

\section{Low-order network model setup}

The numerical setup has been divided into six regions with three jump conditions with pressure losses, one jump condition at the flame and two boundary conditions as shown in Figure 6. The values of the cross-sectional area normalised against the cross-sectional area of the combustion chamber, the lengths of the sections normalised by the reference length and the temperature normalised by the temperature of the air at the inlet for each section are listed in Table 2. The jump matrices to connect acoustic waves between sections are calculated using Equation 15 and Equation 16. The reflection coefficient of the inlet is taken $R_{\text {inlet }}$. The outlet reflection coefficient $R_{\text {out }, 1}$ is measured experimentally using multimicrophone technique. Several values of the outlet reflection coefficient around the measured one are
Figure 5. Numerically computed FTF and the FTF modelled with Equation (11). 


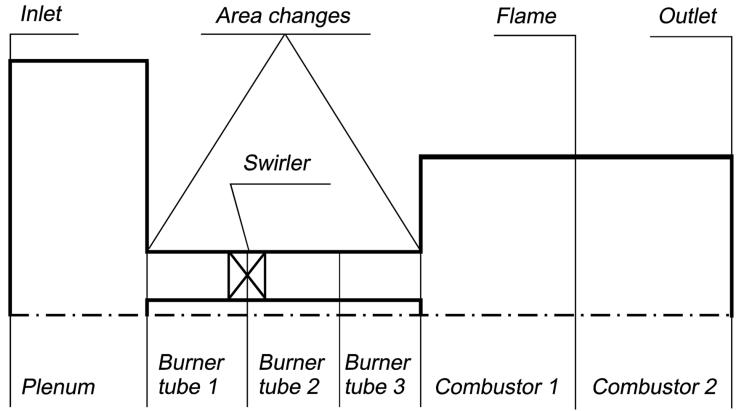

Figure 6. Scheme of network model numerical setup divided into sections.

tioned at $x_{f}=0.1$. This value corresponds to the maximum of the heat release in the longitudinal direction in LES. Mean temperature is uniform in the first five sections. The temperature gradient coincides with the position of the active flame and is situated between Sections 5 and 6 .

\section{Results of linear network model simulations}

For each set of parameters, the simulation is run for $0.3 \mathrm{~s}$ that is enough to observe whether the pressure oscillations grow or decay in time. The setup is excited at the inlet for first $t_{\text {exc }}=0.1 \mathrm{~s}$ by the broadband excitation in the range of Strouhal number $0 \div 7.53$ with the maximum amplitude of the signal $5 \mathrm{~Pa}$. After $0.1 \mathrm{~s}$ till $0.3 \mathrm{~s}$, the system is left to evolve by itself without external excitations. We make use of a parameter called cycle increment that gives information about the thermoacoustic stability of the system. It is possible to calculate the cycle increment from the time-domain simulations assuming the following law for the pressure perturbations:

$$
p^{\prime}(t)=\sum_{i=1}^{n} P_{i} \sin \left(2 \pi f_{i}+\theta_{i}\right) e^{\alpha_{i}\left(t-t_{e x c}\right)},
$$

Table 1. Normalised values of parameters $\tau_{i}$ and $\sigma_{i}$ for the FTF model (11).

\begin{tabular}{|c|c|c|}
\hline$i$ & $\boldsymbol{\tau}_{\boldsymbol{i}}[-]$ & $\boldsymbol{\sigma}_{\boldsymbol{i}},[-]$ \\
\hline 1 & 0.37 & 0.18 \\
\hline 2 & 0.69 & 0.11 \\
\hline
\end{tabular}

Table 2. Values of normalised parameters imposed in the network model.

\begin{tabular}{|c|c|c|c|c|}
\hline$N$ & Section & Area & Length & Temperature \\
\hline 1 & Plenum & 1 & 0.78 & 1 \\
\hline 2 & Burner tube 1 & 0.15 & 0.015 & 1 \\
\hline 3 & Burner tube 2 & 0.15 & 0.024 & 1 \\
\hline 4 & Burner tube 3 & 0.15 & 0.030 & 1 \\
\hline 5 & Combustor 1 & 1 & $x_{f l}=0.1$ & 2.65 \\
\hline
\end{tabular}



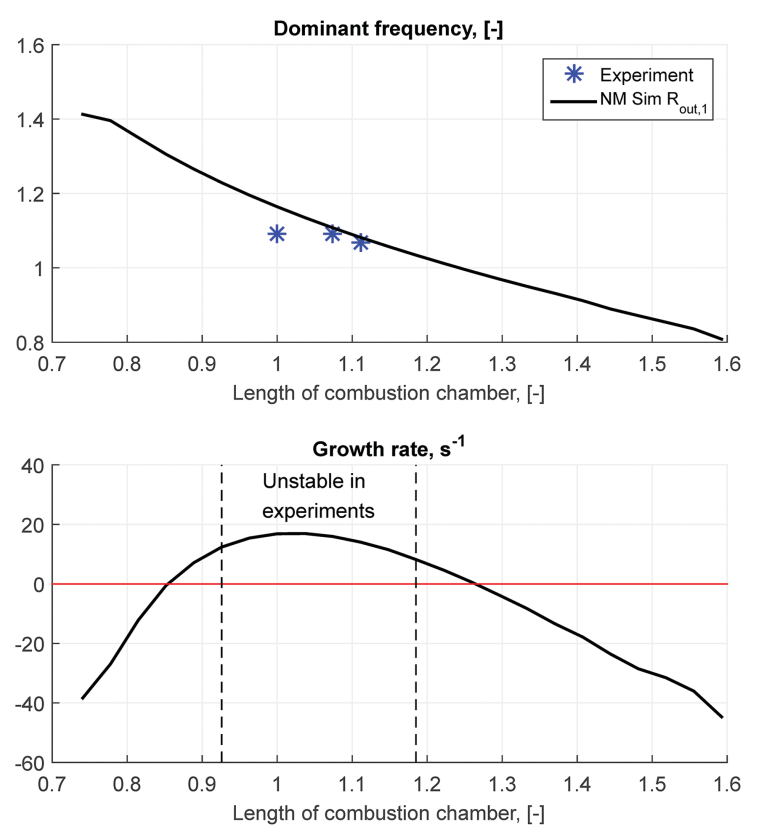

Figure 7. Dominant frequencies of the pressure oscillations and their cycle increments for various lengths of the combustion chamber.

where $f_{i}$ is one of the frequencies of pressure oscillations after $t_{\text {exc }}, n$ is the number of the frequencies of pressure oscillations after $t_{\text {exc }}, P_{i}$ is the amplitude of pressure oscillations at $f_{i}$ at the time $t_{\text {exc }}, \theta_{i}$ is the phase of the pressure oscillations at $f_{i}$ and $\alpha_{i}$ is the cycle increment of the mode $f_{i}$. Positive values of the cycle increment parameter $\alpha_{i}$ indicate that the system is unstable, and the negative values of $\alpha_{i}$ mean that the system is stable.

The frequencies of oscillations and their cycle increments are computed by approximating the time history of the pressure oscillations by Equation (17) using the least-squares method. In the simulations presented in this study, either one or none unstable frequency per run is detected, thus $n=1$ for all simulations in the network model.

Dominant frequencies of pressure oscillations and their cycle increments for various lengths of the combustion chamber are shown in Figure 7. Frequencies are normalised with respect to the mean flow velocity at the burner exit and the diameter of the combustion chamber. The dominant frequency of oscillations decreases while increasing the length of the combustion chamber. This indicates the acoustic nature of the modes computed in this study. The calculated frequencies agree well with the experimentally observed frequencies.

Thermoacoustic instabilities were observed in experiments in the range of combustion chamber length $L_{\text {c.c. }}=0.93 \div 1.19$. Simulations in the network model capture the unstable behaviour of the system qualitatively and predict the setup to be unstable for the slightly wider range of the values of the normalised combustion chamber length in $L_{c . c \text {. }}=0.85 \div 1.30$ (see Figure 7).

\section{Results of weakly nonlinear network model simulations}

It is possible to perform a weakly nonlinear analysis using the network model and the nonlinear heat release model. To do so, it is necessary to know the FDF - the response of the heat release to acoustic perturbations of different frequencies and different amplitudes. Since there is no available computed FDF of the setup, it is possible to mimic the nonlinear flame dynamics assuming the dependence of the parameters $\tau_{i}$ and $\sigma_{i}$ on the normalised amplitude of velocity perturbations $A$ upstream the flame. It was observed in a laboratory setup (Iurashev et al., 2016a) that while increasing the amplitude of velocity excitation, the peak of the heat release distribution along the longitudinal axis was shifted towards the burner and the heat release distribution along the longitudinal axis became wider, i.e., the flame length was augmented. This means that when the amplitude of velocity perturbations upstream the flame is increasing, parameters $\tau_{i}$ are decreasing and parameters $\sigma_{i}$ are increasing:

$$
\begin{gathered}
\tau_{1}=0.37-0.16 A^{2}, \\
\tau_{2}=0.69-0.16 A^{2}, \\
\sigma_{1}=0.18+0.07 A, \\
\sigma_{2}=0.11+0.07 A .
\end{gathered}
$$

Square decay of $\tau_{i}$ parameters and linear increase of $\sigma_{i}$ parameters gave smaller norms of residuals in Iurashev et al. (2016a) and are used in this study as well. Ratios of the second terms to the first terms of Equations (19a) and (20a) are taken the same as in Iurashev et al. (2016a). Absolute values of the second terms in Equations (19b) and (20b) are the same as in Equations (19a) and (20a), respectively. 


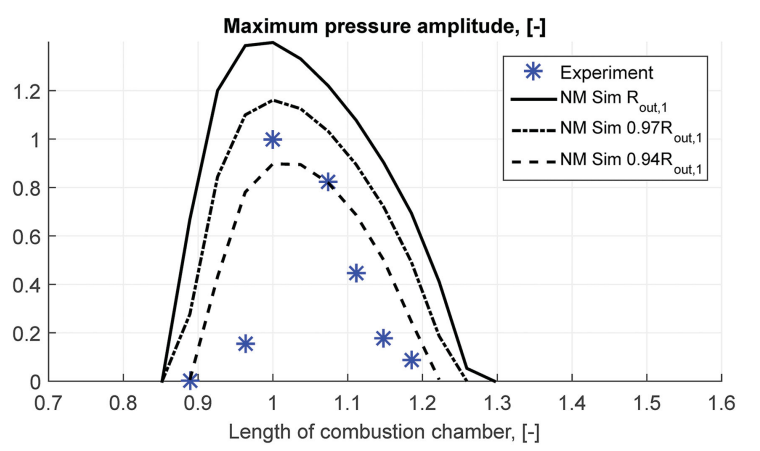

Figure 8. Amplitude of pressure oscillations for various values of the combustion chamber length. combustion chamber length that are predicted to be unstable by the linear analysis and for three values of the outlet reflection coefficient $R_{\text {out }, 1}, 0.97 R_{\text {out }, 1}$, and $0.94 R_{\text {out }, 1}$.

Unstable frequencies of the pressure oscillations in the weakly nonlinear analysis are the same as in the linear analysis shown in Figure 7. Nonlinear simulations capture well the trend of experimental dependence of the pressure oscillations amplitude on the length of the combustion chamber (see Figure 8) with the assumed nonlinear model of the FDF. Simulations employing the outlet reflection coefficient reduced by $6 \%$ with respect to the measured value give a good match with experimental data.

\section{Conclusions and current work}

In this study, a three-step analysis of combustion instabilities in the time domain has been applied to an industrial atmospheric test rig. The first step consists in obtaining the FTF of the system by means of LES. The second step is to approximate the FTF obtained in the first step with the time-lag-distributed model for the technically premixed swirl-stabilised flames. The third step is to perform time-domain simulations using wave-based approach implemented in Simulink with the FTF from the second step.

The results of simulations using the three-step method show that it can be used successfully for the stability analysis. Simulations are run in the same range of the combustion chamber length as in the experiments. The unstable frequencies computed in the simulations match the unstable frequencies observed in the experiments. Simulations predict setup to be unstable in a slightly wider range of combustion chamber length than in the experiments, probably because of uncertainties in the outlet reflection coefficient measurements.

The amplitudes of pressure oscillations computed with the nonlinear analysis for different values of combustion chamber length capture the behaviour observed in experiments.

Currently, less costly Unsteady Reynolds-Averaged Navier-Stokes (URANS) simulations are being performed to compute the FTF for larger number of frequencies and to calculate the FDF of the setup. A future development of this study is to take into consideration of entropy waves propagation.

\section{Acknowledgements}

Authors would like to acknowledge Prof. Maria Heckl for a smooth running of the TANGO project and for many useful discussions. Authors are thankful to Prof. Alessandro Bottaro for his suggestions in this work.

\section{Funding sources}

The presented work is part of the Marie Curie Initial Training Network Thermo-acoustic and aeroacoustic nonlinearities in green combustors with orifice structures (TANGO). We gratefully acknowledge the financial support from the European Commission under call FP7-PEOPLE-ITN-2012. 


\section{Competing interests}

Dmytro Iurashev declares that he has no conflict of interest. Giovanni Campa declares that he has no conflict of interest. Vyacheslav Anisimov declares that he has no conflict of interest. Ezio Cosatto declares that he has no conflict of interest. Luca Rofi declares that he has no conflict of interest. Edoardo Bertolotto declares that he has no conflict of interest.

\section{References}

Albayrak A., Blumenthal R. S., Ulhaq A., and Polifke W. (2015). An analytical model for the impulse response of laminar premixed flames to equivalence ratio perturbations. Proceedings of the Combustion Institute. 36 (3): 3725-3732. https://doi. org/10.1016/j.proci.2016.06.002.

Bellucci V., Schuermans B., Nowak D., Flohr P., and Paschereit C. O. (2005). Thermoacoustic modeling of a gas turbine combustor equipped with acoustic dampers. Journal of Engineering for Gas Turbines and Power. 127 (2): 372-379. https:// doi.org/10.1115/1.1791284.

Bothien M. R., Noiray N., and Schuermans B. (2015). Analysis of azimuthal thermo-acoustic modes in annular gas turbine combustion chambers. Journal of Engineering for Gas Turbines and Power. 137 (6): 061505-1-8. https://doi.org/10.1115/ 1.4028718 .

Camporeale S. M., Fortunato B., and Campa G. (2011). A finite element method for three-dimensional analysis of thermoacoustic combustion instability. Journal of Engineering for Gas Turbines and Power. 133 (1): 011506. https://doi. org/10.1115/1.4000606.

Colin O., Ducros F., Veynante D., and Poinsot T. (2000). A thickened flame model for large eddy simulations of turbulent premixed combustion. Physics of Fluids. 12 (7): 1843-1863. https://doi.org/10.1063/1.870436.

Ćosić B., Moeck J. P., and Paschereit C. O. (2014). Nonlinear instability analysis for partially premixed swirl flames. Combustion Science and Technology. 186 (6): 713-736. https://doi.org/10.1080/00102202.2013.876420.

Dowling A. P. and Stow S. R. (2005). Acoustic analysis of gas turbine combustors in combustion instabilities in gas turbine engines. Progress in Astronautics and Aeronautics, edited by Lieuwen T. C. and Yang V. Washington D.C.: AIAA. 210: $369-414$

Han X., Li J., and Morgans A. S. (2015). Prediction of combustion instability limit cycle oscillations by combining flame describing function simulations with a thermoacoustic network model. Combustion and Flame. 162 (10): 3632-3647. https://doi.org/10.1016/j.combustflame.2015.06.020.

Hermeth S., Staffelbach G., Gicquel L., and Poinsot T. (2013). LES evaluation of non-linear effects on the dynamic flame response in a real gas turbine combustion chamber. ASME Paper GT2013-95699. 1B: V01BT04A052. https://doi.org/ 10.1115/GT2013-95699.

Huang Y. and Yang V. (2009). Dynamics and stability of lean-premixed swirl-stabilized combustion. Progress in Energy and Combustion Science. 35 (4): 293-364. https://doi.org/10.1016/j.pecs.2009.01.002.

Huber A. and Polifke W. (2009a). Dynamics of practical premixed flames, part I: Model structure and identification. International Journal of Spray and Combustion Dynamics. 1 (2): 199-228. https://doi.org/10.1260/175682709788707431.

Huber A. and Polifke W. (2009b). Dynamics of practical premixed flames, part II: Identification and interpretation of CFD data. International Journal of Spray and Combustion Dynamics. 1 (2): 229-250. https://doi.org/10.1260/ 175682709788707440 .

Idelchik I. E. (1992) Handbook of Hydraulic Resistance, 3rd Edition (in Russian), Moscow: Mashinostroienie.

Iurashev D., Campa G., and Anisimov V. V. (2016a). Response of swirl-stabilized perfectly premixed flame to highamplitude velocity excitations. Proceedings of the 23rd International Congress on Sound and Vibration, Athens, Greece.

Iurashev D., Campa G., Anisimov V. V., and Cosatto E. (2016b). Two-step approach for pressure oscillations prediction in gas turbine combustion chambers. International Journal of Spray and Combustion Dynamics. Thermoacoustic Instabilities in Gas Turbines and Rocket Engines: Industry meets Academia. Munich, Germany. Paper No. GTRE-014. https://doi.org/ http://dx.doi.org/10.1177/1756827717711016.

Kim K., Lee J., Quay B., and Santavicca D. (2011). Spatially distributed flame transfer functions for predicting combustion dynamics in lean premixed gas turbine combustors. Combustion and Flame. 157 (9): 1718-1730. https://doi.org/10.1016/j. combustflame.2010.04.016.

Komarek T. and Polifke W. (2010). Impact of swirl fluctuations on the flame response of a perfectly premixed swirl burner. Journal of Engineering for Gas Turbines and Power. 132 (6): 061503-1,7. https://doi.org/10.1115/1.4000127.

Laera D. and Camporeale S. M. (2017). A weakly nonlinear approach based on a distributed flame describing function to study the combustion dynamics of a full-scale lean-premixed swirled burner. Journal of Engineering for Gas Turbines and Power. 139 (9): 091501. https://doi.org/10.1115/1.4036010. 
Li J. and Morgans A. S. (2015). Time domain simulations of nonlinear thermoacoustic behaviour in a simple combustor using a wave-based approach. Journal of Sound and Vibration. 346: 345-360. https://doi.org/10.1016/j.jsv.2015.01.032.

Lieuwen T. C. and Yang V. (2005). Combustion instabilities in gas turbine engines. Progress in Astronautics and Aeronautics Washington. D.C.: AIAA. 210.

Nicoud F., Benoit L., Sensiau C., and Poinsot T. (2007). Acoustic modes in combustors with complex impedances and multidimensional active flames. AIAA Journal. 45 (2): 426-441. https://doi.org/10.2514/1.24933.

Palies P., Durox D., Schuller T., and Candel S. (2011). Nonlinear combustion instability analysis based on the flame describing function applied to turbulent premixed swirling flames. Combustion and Flame. 158 (10): 1980-1991. https:// doi.org/10.1016/j.combustflame.2011.02.012.

Pankiewitz C. and Sattelmayer T. (2003). Time domain simulation of combustion instabilities in annular combustors. Journal of Engineering for Gas Turbines and Power. 123 (3): 677-685. https://doi.org/10.1115/1.1582496.

Poinsot T. J. and Lele S. K. (1992). Boundary conditions for direct simulations of compressible viscous flows. Journal of Computational Physics. 101 (1): 104-129. https://doi.org/10.1016/0021-9991(92)90046-2.

Poinsot T. and Veynante D. (2005) Theoretical and numerical Combustion, 2nd edition. R. T. Edwards Inc. ISBN 9781930217102 .

Rofi L., Campa G., Anisimov V., Daccà F., Bertolotto E., et al. (2015). Numerical procedure for the investigation of combustion dynamics in industrial gas turbines: LES, RANS and thermoacoustics. ASME paper GT2015-42168. 4A: V04AT04A016. https://doi.org/10.1115/GT2015-42168.

Selle L., Lartigue G., Poinsot T., Koch R., Schildmacher K. U., et al. (2004). Compressible large eddy simulation of turbulent combustion in complex geometry on unstructured meshes. Combustion and Flame. 137 (4): 489-505. https://doi. org/10.1016/j.combustflame.2004.03.008.

Schønfeld F. and Rudgyard M. (1999). Steady and unsteady flows simulations using the hybrid flow solver AVBP. AIAA Journal. 137 (11): 1378-1385. https://doi.org/10.2514/2.636.

Schuermans B., Bellucci V., Guethe F., Meili F., Flohr P., et al. (2004). A detailed analysis of thermoacoustic interaction mechanisms in a turbulent premixed flame. ASME paper GT2004-53831.

Schuermans B., Guethe F., Pennell D., Guyot D., and Paschereit C. O. (2010). Thermoacoustic Modeling of a Gas Turbine Using Transfer Functions Measured Under Full Engine Pressure. Journal of Engineering for Gas Turbines and Power. 132 (11): 111503-1-9. https://doi.org/10.1115/1.4000854.

Schuller T., Ducruix S., Durox D., and Candel S. (2002). Modeling tools for the prediction of premixed flame transfer functions. Proceedings of the Combustion Institute. 29 (1): 107-113. https://doi.org/10.1016/S1540-7489(02)80018-9.

Silva C. F., Nicoud F., Schuller T., Durox D., and Candel S. (2013). Combining a Helmholtz solver with the flame describing function to assess combustion instability in a premixed swirled combustor. Combustion and Flame. 160 (9): 1743-1754. https://doi.org/10.1016/j.combustflame.2013.03.020.

Sirignano W. A. (2015). Driving mechanisms for combustion instability. London, Edinburgh and Dublin Philosophical Magazine and Journal of Science. 17: 419-422.

Smagorinsky J. (1963). General circulation experiments with the primitive equations I. The basic experiment. Monthly Weather Review. 91 (3): 99-164. https://doi.org/10.1175/1520-0493(1963)091<0099:GCEWTP>2.3.CO;2.

Staffelbach G., Gicquel L., Boudier G., and Poinsot T. (2009). Large eddy simulation of self-excited azimuthal modes in annular combustors. Proceedings of the Combustion Institute. 32 (2): 2909-2916. https://doi.org/10.1016/j. proci.2008.05.033

Wolf P., Staffelbach G., Gicquel L., Müller J. D., and Poinsot T. (2012). Acoustic and large eddy simulation studies of azimuthal modes in annular combustion chambers. Combustion and Flame. 159 (11): 3398-3413. https://doi.org/10.1016/ j.combustflame.2012.06.016.

\section{Appendix A. Matrices for jump conditions between network model sections}

Matrices for jump conditions between sections for the case of area decrease are

$$
\mathbf{F}_{\text {decr }}=\left[\begin{array}{cc}
\frac{S_{d}}{\bar{c}_{s, d}}\left(1+M_{d}\right) & \frac{S_{u}}{\bar{c}_{s, u}}\left(1-M_{u}\right) \\
\frac{1}{\bar{\rho}_{d}}\left(1+M_{d}\left(1+\zeta_{\text {decr }}\right)\right) & -\frac{1}{\bar{\rho}_{u}}\left(1-M_{u}\right)
\end{array}\right],
$$




$$
\mathbf{K}_{\text {decr }}=\left[\begin{array}{cc}
\frac{S_{u}}{\bar{s}_{s, u}}\left(1+M_{u}\right) & \frac{S_{d}}{\bar{c}_{s, d}}\left(1-M_{d}\right) \\
\frac{1}{\bar{\rho}_{u}}\left(1+M_{u}\right) & -\frac{1}{\bar{\rho}_{d}}\left(1-M_{d}\left(1+\zeta_{\text {decr }}\right)\right)
\end{array}\right],
$$

where $S$ is the cross-sectional area, $M$ is the mean Mach number and $\zeta$ is the pressure loss coefficient. Matrices for jump conditions between sections for the case of area increase are

$$
\begin{gathered}
\mathbf{F}_{\text {incr }}=\left[\begin{array}{cc}
\frac{S_{d}}{\bar{s}_{s, d}}\left(1+M_{d}\right) & \frac{S_{u}}{\bar{c}_{s, u}}\left(1-M_{u}\right) \\
\frac{1}{\bar{\rho}_{d}}\left(1+M_{d}\right) & -\frac{1}{\bar{\rho}_{u}}\left(1-M_{u}\left(1-\zeta_{\text {incr }}\right)\right)
\end{array}\right], \\
\mathbf{K}_{\text {incr }}=\left[\begin{array}{cc}
\frac{S_{u}}{\bar{c}_{s, u}}\left(1+M_{u}\right) & \frac{S_{d}}{\bar{c}_{s, d}}\left(1-M_{d}\right) \\
\frac{1}{\bar{\rho}_{u}}\left(1+M_{u}\left(1-\zeta_{\text {incr }}\right)\right) & -\frac{1}{\bar{\rho}_{d}}\left(1-M_{d}\right)
\end{array}\right],
\end{gathered}
$$

Matrices for jump conditions between sections for the case of temperature jump with active flame and constant cross-sectional area are

$$
\begin{gathered}
\mathbf{J}=\left[\begin{array}{cc}
\left(1+2 M_{d}+M_{d}^{2}\right) & -\left(1-2 M_{u}+M_{u}^{2}\right) \\
{\left[\frac{\bar{c}_{s}+\gamma \bar{u}}{\gamma-1}+\frac{3 \bar{u}^{2}}{2 \bar{c}_{s}}+\frac{\bar{u}^{3}}{2 \bar{c}_{s}^{2}}\right]_{d}} & -\left[\frac{\overline{c_{s}}-\gamma \bar{u}}{\gamma-1}+\frac{3 \bar{u}^{2}}{2 \bar{c}_{s}}-\frac{\bar{u}^{3}}{2 \bar{c}_{s}^{2}}\right]_{u}
\end{array}\right], \\
\mathbf{J}=\left[\begin{array}{ccc}
\left(1+2 M_{u}+M_{u}^{2}\right) & -\left(1-2 M_{d}+M_{d}^{2}\right) & 0 \\
{\left[\frac{\bar{c}_{s}+\gamma \bar{u}}{\gamma-1}+\frac{3 \bar{u}^{2}}{2 \bar{c}_{s}}+\frac{\bar{u}^{3}}{2 \bar{c}_{s}^{2}}\right]_{u}} & -\left[\frac{\bar{c}_{s}-\gamma \bar{u}}{\gamma-1}+\frac{3 \bar{u}^{2}}{2 \bar{c}_{s}}-\frac{\bar{u}^{3}}{2 \bar{c}_{s}^{2}}\right]_{d} & \frac{1}{S}
\end{array}\right],
\end{gathered}
$$

where $\gamma$ is the heat capacity ratio. 